\title{
Multiple Intussusceptions Associated with Meconium Plugs: A Case and Literature Review
}

\author{
Kumar Diwakar, MD*, Essa Al Awad, MD, FRCPE*, Shabih U Hasan, MD, FRCPC and Kamran Yusuf, MD, FAAP \\ Department of Pediatrics, Cumming School of Medicine University of Calgary, Canada \\ *Corresponding author: Essa Al Awad, MD, Section of Neonatology, Department of Pediatrics, Cumming School of Medicine University of Calgary, \\ Alberta, Canada \\ *Kamran Yusuf MD, Section of Neonatology, Department of Pediatrics, Cumming School of Medicine University of Calgary, Rm 2273, Health Sciences \\ Center, 3330 Hospital Drive NW, Calgary, Alberta, T2N 4N1, Canada
}

Submission: 眥 September 26, 2017; Published: 望 November 02, 2017

\begin{abstract}
Intussusception is uncommon in neonates, especially preterm neonates. Even rarer is the presence of multiple intussusceptions. As the classical signs of intussusception are not present in preterm neonates, the diagnosis is often confused with necrotizing enterocolitis, a much more common abdominal pathology. This delay in diagnosis is thought to be responsible for the high rate of perforations and necrosis seen in intussusception in preterm neonates. We describe a 25 week gestation preterm male neonate who presented with jejuno-jejuno and jejuno-ileal intussusceptions. To our knowledge this is only the second reported case of multiple intussusceptions in a preterm neonate. In addition, we provide some clinical guidelines that may help in making an earlier diagnosis.
\end{abstract}

Keywords: Intussusceptio; Preterm neonate; Necrotizing enterocolitis; Multiple; Meconium plugs

\section{Introduction}

Intussusception is a common cause of an acute abdomen in children less than two years old [1] Although most cases occur under the age of one year, it is relatively uncommon in neonates, comprising $3 \%$ of all causes of neonatal intestinal obstruction and $0.3 \%$ of all pediatric intussusceptions [2,3]. As compared to term neonates, intussusception is even rarer in preterm neonates, where the diagnosis is usually delayed as it is often confused with necrotizing enterocolitis (NEC), a much more common abdominal pathology in preterm neonates [2]. Furthermore, almost all cases of neonatal intussusceptions reported in the literature are single intussusceptions [2,4-7]. We describe a 25 week gestation male neonate who had both jejuno-jejuno and jejuno-ileal intussusceptions. To our knowledge this is only the second reported case of multiple intussusceptions in a preterm neonate.

\section{Case Report}

Our case was a 25 week gestation male neonate, born to a 29 years old primigravida by emergency caesarian section because of preterm labour and breech presentation. Till the onset of preterm labour, the pregnancy was uneventful. The infant had a birthweight of $930 \mathrm{gm}$ with Apgar scores of 3 and 5 at 1 and 5 minutes respectively. Due to poor respiratory effort, the infant was endotracheally intubated and given a dose of surfactant. He received antibiotics for 48 hours and was extubated on the second day of life but had to be reintubated the same day for frequent apneas despite being on caffeine. A cranial ultrasound on the third day of life showed a left grade IV intraventricular hemorrhage. On day 4 an echocardiogram showed a small hemodynamically nonsignificant patent ductus arteriosus. The infant was started on trophic feeds on day 4, which he tolerated for 48 hours but then developed hypotension and a dusky looking abdominal wall. Feeds were stopped, antibiotics restarted and the hypotension responded to a normal saline bolus and inotropes. The abdominal x-ray was non-specific with asymmetric bowel gas pattern demonstrating paucity of right lower quadrant gas and no evidence of pneumotosis intestinalis or free air. Over the next 48 hours the infant's condition improved with a normal abdominal examination, he was passing meconium and was restarted on trophic feeds. However, two days later he again developed a dusky looking abdomen and feeds were withheld. The abdominal x-ray was again non-specific with paucity of right lower quadrant gas and no evidence of pneumotosis or free air. An abdominal ultrasound on day 11 of life showed mild thickening and increased echogenicity of bowel wall and mild ascites without any evidence of intussusception.

The infant's condition continued to deteriorate with increasing ventilatory requirement and inotropic support while he continued to be on antibiotics including metronidazole. An abdominal x-ray on the $13^{\text {th }}$ day of life showed free air, necessitating the placement of a 
peritoneal drain, through which meconium was drained. The next day, the infant was stable to undergo a laprotomy which showed the presence of jejuno-jejuno and jejuno-ileal intussusceptions along with two associated perforations. In addition, there was impacted meconium in the small intestine, especially in the terminal ileum. The intussusceptions were reduced and the most proximal perforation was resected with the creation of a jejunostomy and a mucous fistula. The distal perforation was resected and an end to end anastomosis performed. The infant tolerated the surgery well and had an uneventful post-operative course. Despite being critically ill pre-operatively, the infant always had a normal platelet count and at no point during the course of his illness, did he have an abdominal mass or blood per rectum. Screening for cystic fibrosis was negative.

\section{Discussion}

There have been three reports of multiple intussusceptions in neonates, one in a preterm and two in term neonates. Slam et al described a 25 weeks gestation male neonate who presented at 39 days of life with abdominal distention and feeding intolerance [8]. A laparotomy at 53 days of life showed six ileo-ileal intussusceptions. This infant, like our case, had normal platelet counts, paucity of air in the right lower quadrant on abdominal films and meconium plugs. The infant died 10 days after surgery due to sepsis. Singh et al reported a 10 day old term male neonate with ileo-ileal and ileo-colic intussusceptions [9]. Pre-operatively, this infant had an episode of bleeding per rectum and also a palpable mass in the right lower quadrant. In addition, abdominal ultrasound showed the target sign. This infant also died five days after surgery due to septicemia. Uecki et al [10] described a term male neonate with transposition of the great arteries who underwent atrial septostomy on the second day of life $[7,10]$. On the third day of life the infant developed abdominal distention and massive bloody stools. Abdominal ultrasound was negative but exploratory laparotomy showed ileo-ileal and ileo-colic intussusceptions. This infant died of cardiac complications post-operatively. Interestingly, intussusception in children and neonates has a male preponderance and all cases of multiple intussusceptions in neonates, including ours, have been males [2,5,7-11].

Intussusception in preterm neonates is usually misdiagnosed as NEC, leading to delay in diagnosis, as in our case, of a median of seven days from the onset of symptoms [2,4]. This delay in diagnosis is responsible for the high rates of intestinal perforation and necrosis in preterm infants with intussusception $[2,5]$. The classical clinical features of intussusception such as an abdominal mass, blood in the stools and vomiting are uncommon in preterm neonates and the diagnosis is usually made intra-operatively [2,46]. In addition, these and other signs such as abdominal distention and gastric residuals which may be present in intussusception are also present in NEC, a much more common condition in preterm neonates, the treatment of which, at least initially, is conservative, further delaying the diagnosis $[2,4,5]$.

Unlike older children, imaging modalities such as plain abdominal films, contrast enemas and abdominal ultrasounds are not very helpful in making a diagnosis of intussusception in preterm infants [2,4-6]. Lack of bowel gas in the right lower quadrant, as in our case, has been described in other preterm infants with intussusception but is an inconsistent finding (Figure 1) $[1,8,12]$. Although contrast enemas may be both diagnostic and therapeutic in older infants, they are not helpful in preterm infants as intussusception in preterm infants is almost always confined to the small intestine, unlike older infants where it is usually ileocolic $[5,6,8,12]$. Some investigators have in fact recommended that contrast enemas should be avoided in preterm infants, as they increase the risk for perforation [5,6]. In older infants, abdominal ultrasound has a sensitivity of over $98 \%$ and specificity of $100 \%$ in the diagnosis of intussusception [11,12]. In our review of intussusception in preterm infants, of the 35 or so reported cases, only two were diagnosed with abdominal ultrasound [2]. The lack of efficacy of abdominal ultrasound in diagnosing intussusception in preterm infants may again be due to the absence of colonic involvement in preterm infants $[4,11]$. However, ultrasound is an easy to use, non-invasive, bedside investigation and in an effort to make an early diagnosis, should be considered in any neonate suspected to have intussusception [2].

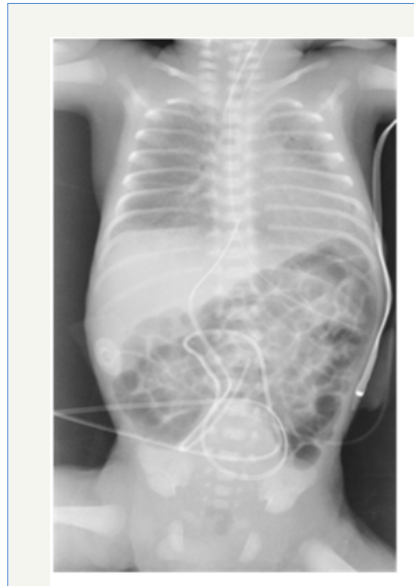

(A)

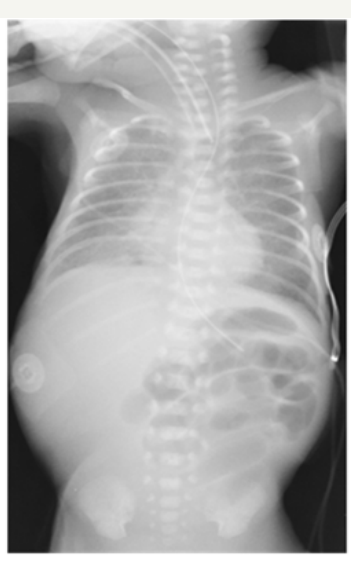

(B)
Figure 1: Abdominal $x$-ray of the infant.

(A) Day two of life demonstrating a symmetrical gas pattern.

(B) Day 10 of life showing an asymmetrical gas pattern with paucity of gas in the right lower quadrant.

The etiology of intussusception in preterm infants is largely unknown. While a lead point for the intussusception may be identified in older and term infants, it is uncommon in preterm infants [3]. Mooney et al [6] in a review of ten cases of intussusception in preterm infants identified a lead point in two cases, both with an atretic intestinal segment [6]. Gorgen-Pauly [5] reviewed seventeen cases of intussusception in preterm infants with four having a potential lead point [5]. There, however, have been two reported cases where intussusception in preterm infants was associated with meconium plugs. Glick et al described a 28 week gestation male infant who along with meconium plugs also had an ileo-ileal intussusception [13]. In the case reported by Slam, multiple intussusceptions were associated with meconium 
plugs and lymphoid hyperplasia of the intestines which could have served as the lead point [8]. In older children with cystic fibrosis, intussusception secondary to inspissated feces in the terminal ileum is well described [11]. Although cystic fibrosis screening in our patient was negative, meconium plugs could have served as the lead points for the multiple intussusceptions.

\section{Conclusion}

In summary, we report only the second case of multiple intussusceptions in a preterm infant. Although intussusception in preterm infants is uncommon, it is easily confused with NEC, leading to delay in diagnosis. Even rarer is the presence of multiple intussusceptions. Although overlapping with the clinical findings in NEC, the presence of a male gender, paucity of bowel gas in the right lower quadrant on an abdominal x-ray and a normal platelet count in a sick neonate with suspected NEC, should alert the physician to the possibility of intussusception. Importantly, if one intussusception is discovered at surgery, others should also be looked for, especially if there are meconium plugs.

\section{References}

1. Pepper VK, Stanfill AB, Pearl RH (2012) Diagnosis and management of pediatric appendicitis, intussusception, and Meckel diverticulum. Surg Clin North Am 92(3): 505-526.

2. Biarge MM, Garcia-Alix A, del Hoyo ML, Alarcon A, de Pipaon MS, et al. (2004) Intussusception in a preterm neonate; a very rare, major intestinal problem - systematic review of cases. J Perinat Med 32(2): 190-194.
3. Wang NL, Yeh ML, Chang PY, Sheu JC, Chen CC, et al. (1998) Prenatal and neonatal intussusception. Pediatr Surg Int 13(4): 232-236.

4. Avansino JR, Bjerke S, Hendrickson M, Stelzner M, Sawin R (2003) Clinical features and treatment outcome of intussusception in premature neonates. J Pediatr Surg 38(12): 1818-1821.

5. Gorgen-Pauly U, Schultz C, Kohl M, Sigge W, Moller J, et al. (1999) Intussusception in preterm infants: case report and literature review. Eur J Pediatr 158(10): 830-832.

6. Mooney DP, Steinthorsson G, Shorter NA (1996) Perinatal intussusception in premature infants. J Pediatr Surg 31(5): 695-697.

7. Ueki I, Nakashima E, Kumagai M, Kimura A, Hashimoto T (2004) Intussusception in neonates: analysis of 14 Japanese patients. J Paediatr Child Health 40(7): 388-391.

8. Slam KD, Teitelbaum DH (2007) Multiple sequential intussusceptions causing bowel obstruction in a preterm neonate. J Pediatr Surg 42(7): 1279-1281.

9. Singh JK, Bawa M, Kanojia RP, Ghai B, Menon P, et al. (2009) Idiopathic simultaneous intussusceptions in a neonate. Pediatr Surg Int 25(5): 445447.

10. Ueki I, Nakashima E, Kumagai M, Kimura A, Hashimoto T (2005) Two neonates with intussusception accompanying severe hypoxia. Pediatr Int 47(5): 595-597.

11. Robb A, Lander L (2008) Intussusception in infants and young children. Surgery 26: 291-293.

12. Williams H (2008) Imaging and intussusception. Arch Dis Child Educ Pract 93: 30-36.

13. Glick B, Alpan G, Vinograd I, Udassin R, Mogle P, et al. (1985) Meconium plugs and intussusception in a premature infant. Am J Perinatol 2(2): 67-69. 